\title{
Trivium
}

Revue franco-allemande de sciences humaines et sociales - Deutsch-französische Zeitschrift für Geistesund Sozialwissenschaften

33 | 2021

Concepts historiques fondamentaux - Démocratie

\section{La « démocratie » dans le mouvement moderne}

\section{Werner Conze}

Traducteur : Anthony Andurand

\section{Q OpenEdition}

\section{Journals}

Édition électronique

URL : https://journals.openedition.org/trivium/7663

DOI : $10.4000 /$ trivium.7663

ISSN : 1963-1820

Éditeur

Les éditions de la Maison des sciences de l'Homme

Référence électronique

Werner Conze, «VI. La « démocratie » dans le mouvement moderne», Trivium [En ligne], 33 | 2021, mis en ligne le 07 juin 2021, consulté le 10 juin 2021. URL : http://journals.openedition.org/trivium/7663 ; DOI : https://doi.org/10.4000/trivium.7663

Ce document a été généré automatiquement le 10 juin 2021

\section{(c) (i) () $\Theta$}

Les contenus des la revue Trivium sont mis à disposition selon les termes de la Licence Creative Commons Attribution - Pas d'Utilisation Commerciale - Pas de Modification 4.0 International. 


\title{
VI. La « démocratie » dans le mouvement moderne
}

\author{
Werner Conze
}

Traduction : Anthony Andurand

\section{NOTE DE L'ÉDITEUR}

p. 873-895 de l'article original

\section{Le «principe démocratique » et la monarchie constitutionnelle}

1 Les sections précédentes ont permis de montrer comment le concept de démocratie, qui pouvait être défini de manière relativement claire dans le cadre de sa tradition et de sa réception alors qu'il n'était jamais tout à fait univoque, a quitté la tour d'ivoire de l'érudition pour être jeté sur le champ de bataille politique révolutionnaire. Le mot "démocratie » en était ainsi venu à désigner un parti politique, dans un but d'autoaffirmation ou pour le dénigrer. De surcroit, la démocratie devait être réalisée d'une nouvelle manière, elle devait être généralisée, autrement dit, ne pas se limiter à des exceptions, à des formes particulières dans un espace restreint, ou à l'inverse, ses prétentions à se réaliser devaient de nouveau être réfutées. Dans ce dernier cas, le discours traditionnel selon lequel la démocratie - au sens classique de la démocratie directe - est irréalisable dans les États de grande taille et fortement peuplés ne suffisait plus, puisqu'avec la création de l'expression de « démocratie représentative » une étape décisive avait été franchie permettant d'appliquer le concept de démocratie aux constitutions modernes. Le principe d'une représentation $\mathrm{du}$ «peuple» institutionnalisée sur le plan constitutionnel rendait a priori possible, pour tous les États, l'identité des gouvernants et des gouvernés. Dans la mesure où (depuis Fichte et Schlegel) une telle constitution a été appelée "démocratie», une telle conviction 
politique « démocratisme » et le mouvement conduit de manière consciente en vue de cet objectif "démocratisation", la voie était ouverte à une expansion et à une utilisation souvent contradictoire du concept. Toute compréhension de la démocratie peut très bien se fonder sur l'idée d'une direction unique ancrée dans la philosophie de l'histoire, comme ce fut d'abord le cas dans le « républicanisme » de Kant. Cependant, les objectifs et les moyens d'y parvenir, dès les premières décennies du XIX ${ }^{\mathrm{e}}$ siècle, étaient à chaque fois définis de manière si différente et le pouvoir de suggestion du terme " démocratie " s'accrut d'une manière si extraordinaire que Guizot, encore sous l'impression de la Révolution de 1848, pouvait le décrire comme «le mot souverain, universel », brandi par tous les partis comme un talisman. [874] «Tel est l'empire du mot démocratie que nul gouvernement, nul parti n'ose vivre, et ne croit le pouvoir, sans inscrire ce mot sur son drapeau ${ }^{296}$. »

2 De fait, entre 1789 et 1848 , le terme "démocratie " fut peu à peu attribué à tout mouvement ou toute idéologie moderne ou s'adaptant à la modernité, ou qui pouvait établir un lien avec eux : à la liberté (constitutionnelle et individuelle) (le libéralisme), à l'égalité comprise comme égalité politique ou entre les hommes (jusqu'au socialisme et au communisme), à la nation et au mouvement national, au christianisme (de quelque manière qu'il soit conçu), et même à la monarchie et au monarchisme. Pour toutes ces tendances majeures, chargées de valeurs, des époques révolutionnaires de l'Europe occidentale et centrale, on peut cependant aussi observer, dans chaque cas, des réactions défensives typiques contre la « démocratie ». À l'attrait du terme répondirent toujours, dans l'Allemagne d'après 1848 et plus fortement encore à partir de 1871, des réticences persistances, voire une certaine hostilité. La préservation, et parfois même la réaffirmation des institutions et des valeurs monarchiques et aristocratiques traditionnelles ont eu pour effet que le terme de démocratie, jusqu'en 1918, n'a jamais pu servir à désigner des constitutions allemandes, excepté dans un regard historique rétrospectif ou dans une perspective d'attente concernant l'avenir. C'est ainsi que, dans l'Allemagne du XIX ${ }^{e}$ siècle, on n'est pas arrivé à cet « empire du mot démocratie » que Guizot avait constaté en 1849 dans le cas de la France, bien que, dans les années 40 et durant la Révolution de 1848/49, la contribution allemande à l'expansion du concept ait été considérable.

Dans les années qui suivirent immédiatement 1815, lorsqu'il s'agissait d'arriver à instaurer un «État constitutionnel» fondé sur la représentation, le terme de " démocratie » - ou mieux, les notions d'« éléments » et de " principes » démocratiques - s'imposèrent quand on devait désigner la tendance à une plus forte participation de parties plus grandes du peuple dans la constitution de l'État. Un lien s'établit entre cet usage et l'idée libérale et romantique d'une liberté germanique ancienne, qui avait été corrompue par le "féodalisme », le terme de "démocratie " se prêtant désormais à une explication de ce qu'elle était.

\section{a) Relier les principes : Görres, Rotteck}

4 Görres incarne de manière emblématique la possibilité, un temps forte, mais finalement avortée, de définir le nouveau type de la monarchie constitutionnelle au moyen du "principe démocratique». Il s'agissait pour Görres de prévenir la menace d'une séparation entre le principe monarchique et le principe démocratique comme deux forces ennemies, de mettre fin à leur désunion et de les associer de manière organique, 
persuadé aussi qu'il était de la nécessité d'une médiation entre les tendances historiques et les tendances rationnelles progressistes de l'époque ${ }^{297}$. Le principe démocratique, issu de l'individuation et de la multitude dans un mouvement allant de bas en haut, devait aller à la rencontre du principe monarchique agissant de haut en bas dans l'esprit d'un tout politique formant une unité. La constitution médiévale qui vit se former le "système féodal ", "lorsque les officiers impériaux s'unirent à la démocratie des propriétaires pour former une aristocratie armée ${ }^{298}$ ", [875] devait servir de modèle. Au-delà de la " pesanteur excessive » d'un absolutisme centralisateur, d'une part, et d'un despotisme jacobin, d'autre part, Görres voulait ainsi faire en sorte qu'une constitution idéale du futur reposât sur une démocratie, avant tout sur celle des propriétaires terriens chargés de faire coöncider une obéissance à la démocratie avec leur propre responsabilité, issue, dans un mouvement de bas en haut, de la communauté. "Une fois que la démocratie aura réussi à se débarrasser de ce despotisme formel, qu'elle se sera de nouveau enracinée dans le vieux sol, qu'elle y aura retrouvé force et vie et les aura transmises à la monarchie affaiblie [...], le mouvement [...] dans la constitution [...] sera de nouveau ascendant ${ }^{299}$. »

5 Proche de la démocratie « historique » de Görres, on trouve Ernst Moritz Arndt. À la suite des abus jacobins, qui avaient fait du terme « une sorte de mort-aux-rats, pour le cœur et les oreilles de tout homme de bien ", Arndt cherchait à retrouver dans la mémoire historique une démocratie pour le présent.

« Tout peuple des constitutions à la fois représentatives et fondées sur des groupes statutaires [Stände], composées de toutes les classes de la population, possède déjà, pour cette raison même, des constitutions démocratiques. Car là où les paysans et les bourgeois des villes, qui forment la partie la plus vaste et la plus respectable du peuple, sont représentés publiquement, on peut parler d'une constitution démocratique ${ }^{300}$.»

$6 \mathrm{Si}$, malgré l'accent mis sur un rééquilibrage, la balance penchait encore chez Görres vers la dimension «historique» du "principe démocratique», au détriment de la dimension fondée sur la raison, la tendance devait s'inverser dans le Manuel de droit constitutionnel [Konstitutionnelles Staatsrecht] (1824) d'Aretin, continué par Rotteck $(1839)^{301}$. Dans cet ouvrage, les auteurs percevaient

«au sein du peuple, soit la totalité de la population [...], trois intérêts principaux, que l'on nomme aussi, dans le langage académique, "principes" ou "éléments": l'intérêt monarchique, l'intérêt aristocratique et l'intérêt démocratique. Ce dernier ne serait pas uniquement représenté par les classes populaires inférieures, mais aussi par toutes les professions qui ne sont pas liées à l'appartenance d'origine, à la propriété et à l'esprit des corporations : les commerçants, les propriétaires de biens mobiliers, les savants et les artistes, les industriels, c'est-à-dire, d'une manière générale, tous les éléments au sein desquels prédomine le désir d'activité, d'éducation et de développement. »

Dans cette vision du droit constitutionnel et de ce que devaient être, de façon optimale, les nouvelles constitutions de l'époque, "l'intérêt démocratique » exigeait les libertés "politiques» et "civiles» habituellement en vigueur dans le droit constitutionnel libéral. Le principe démocratique était donc essentiellement porté par le corps social intermédiaire [Mittelstand] - qui n'apparaissait pas sous ce terme dans l'ouvrage -, celui de la culture et de la propriété, et il devait accorder aux revendications du libéralisme l'importance nécessaire "dans l'essence de ce qu'est la monarchie constitutionnelle, qui, avec sagesse, réunit ces trois intérêts principaux et fait qu'ils se limitent réciproquement ${ }^{302}$ \%. L'harmonisation des principes était ainsi poussée si loin que non 
seulement la monarchie et la démocratie, [876] mais aussi l'aristocratie correspondant aux premières chambres - étaient compatibles ${ }^{303}$. Le fait que la démocratie, dans cette métamorphose moderne de l'ancienne théorie de la constitution mixte, soit intégrée comme un principe s'opposait totalement à une compréhension plus cohérente du constitutionnalisme, telle que Rotteck la décrit lui-même: le principe aristocratique "constitue cependant une pure négation du principe démocratique, tout comme celui-ci est l'ennemi mortel du premier ${ }^{304}$ ». Quelle que soit la façon de délimiter le principe démocratique par rapport au principe aristocratique, plusieurs éléments étaient incontestés dans cette vision de la monarchie constitutionnelle: les tendances monarchiques et démocratiques de l'époque devaient être réconciliées dans un esprit libéral - fondé sur l'histoire et/ou le droit rationnel. La démocratie comprise au sens libéral, en tant que concept de mouvement, renvoyait à un objectif de progrès, qui se voyait cependant limité du fait de compromis avec les « éléments » traditionnels.

7 Ce fut la base du sentiment de supériorité du Sud de l'Allemagne, fondé sur les constitutions d'après 1815. En 1838, Heinrich von Gagern mettait par exemple en exergue «les tendances démocratiques de l'Allemagne du Sud», par contraste avec la Prusse $^{305}$. Lorsqu'on le dissociait du constitutionnalisme et que l'on se référait aux réformes de 1807/1823, "l'élément démocratique " pouvait en revanche s'appliquer à la Prusse. En 1834, David Hansemann mettait notamment en exergue, par contraste avec la France ou les États constitutionnels de l'Allemagne, l'« affaiblissement du pouvoir aristocratique", le "développement du pouvoir des classes populaires inférieures", l'abaissement du cens lors des élections communales, l'« armement du peuple», perçus comme autant d'éléments «démocratiques». Comparé aux États constitutionnels, "la tendance démocratique du gouvernement", ainsi que les éléments et les vues démocratiques de la plupart des fonctionnaires prussiens ne lui paraissaient faire aucun doute ${ }^{306}$. Dans ce pays, l'alliance définie par Hardenberg - « des fondements démocratiques au sein d'un gouvernement monarchique ${ }^{307}$ » - était à nouveau inscrite dans la conscience sociale moderne d'une "classe moyenne » issue d'une "Prusse rhénane» attentive aux questions politiques sous l'influence de la révolution de Juillet. La «tendance démocratique » définie par Hansemann tire son acuité du fait qu'elle se démarque non seulement de l'« aristocratie » agonisante, mais aussi de l'idée politique formelle d'une démocratie représentative et constitutionnelle. La démocratie, comme "élément " ou "tendance ", nullement défendue dans une perspective absolue ou totale, acquiert ainsi, comme chez Tocqueville, un caractère fondamentalement social. Cette approche préfigurait la distinction établie un peu plus tard entre la démocratie ou la révolution purement politique et la démocratie ou la révolution pleinement sociale.

\section{b) Dissocier les principes : Gentz, Schleiermacher, Hegel}

8 [877] En fin de compte, l'adaptation du concept de démocratie à la réalité constitutionnelle allemande ne put s'imposer, du fait que le pouvoir de séparation des « principes » fut placé au premier plan. Ce ne sont pas les médiateurs, comme Aretin et Rotteck, mais les hommes d'État et les idéologues de la "contre-révolution », comme Metternich et Gentz, qui firent obstacle à la jonction des "principes », dans l'esprit de l'article 57 de l'acte final du Congrès de Vienne, stipulant que «le pouvoir politique dans son ensemble devait rester concentré entre les mains du chef de l'État». En 
distinguant les "constitutions territoriales fondées sur des états" [landständische Verfassungen] et les "constitutions représentatives» (1819), Gentz exclut l'élément démocratique de la constitution appelée à être en vigueur dans chaque État allemand, chacune d'entre elles devant être conforme aux formes constitutionnelles de la Confédération germanique. Selon lui, tandis que les "constitutions territoriales" étaient faites pour que "les membres ou les députés exercent [...] un droit de participation à la législation de l'État en vertu de l'existence autonome de certains corps ", les " constitutions représentatives » étaient "fondées sur la fausse idée d'une souveraineté populaire suprême ». Ces dernières substituaient ainsi « le fantôme d'une prétendue liberté du peuple (c'est-à-dire l'arbitraire général) [...] et l'illusion d'une égalité générale des droits, ou ce qui ne vaut pas mieux, d'une égalité générale devant le droit, aux différences intangibles de statut et de droits établies par Dieu lui-même ", qui seraient encore garanties dans les «constitutions territoriales ${ }^{308}$ ». Bien qu'il ne la nommât pas, Gentz visait la démocratie, la volonté de Metternich étant de l'éliminer du droit constitutionnel allemand ${ }^{309}$. Dans cette situation, pour la Prusse également, la « démocratie » restait une notion extraconstitutionnelle : «les idées démocratiques et représentatives, [...] l'agitation démocratique et révolutionnaire et la constitutionnalité de l'Allemagne du Sud » devaient continuer à être « dépopularisées » et exclues ${ }^{310}$. Dans une lettre adressée à Niebuhr le 22 avril 1819, Wilhelm von Humboldt désignait la propriété foncière et les corporations comme «les plus sûrs appuis contre le démocratisme ${ }^{311}$ ». Quelques années auparavant, Schleiermacher avait théorisé historiquement la tendance consistant à considérer la démocratie comme inconciliable avec la réalité constitutionnelle moderne. Schleiermacher transposa dans la construction d'une trajectoire universelle de l'histoire constitutionnelle la théorie traditionnelle selon laquelle la démocratie n'était possible que dans les entités de taille réduite. La démocratie - fût-elle non pas exclusivement, mais éventuellement transformée dans une perspective monarchique ou aristocratique - se trouvait assimilée par lui au caractère politique primitif de la constitution propre à une tribu, tandis que les États modernes présentant une « organisation supérieure » ne pouvaient être que monarchiques, après que la forme aristocratique intermédiaire que représentent les «puissances moyennes » eut été selon lui historiquement dépassée ${ }^{312}$. [878] Schleiermacher renversait ainsi l'idée révolutionnaire d'un mouvement nécessaire de l'histoire vers la démocratie, fréquemment reprise ou développée depuis l'Essai sur le concept de républicanisme ${ }^{313}$ de Schlegel (1796), en opposant l'expérience historique (y compris la Révolution française) aux attentes utopiques pour l'avenir. Les déductions historico-empiriques de Schleiermacher, pour le présent et le futur, l'amenèrent à confirmer que l'État moderne de grande taille, « qui considère l'unité du peuple dans son ensemble comme une unité naturelle, véritable et nécessaire, et l'exprime dans les formes de la vie », ne pouvait être que monarchique, la démocratie étant, quant à elle, renvoyée ad absurdum.

9 Le jugement développé dans la Brockhaus (1818) montre que l'aversion à l'égard de l'utilisation du concept de démocratie dans la théorie constitutionnelle - y compris lorsqu'il était déjà question de la démocratie "représentative » - était très répandue : la démocratie «sombre inexorablement dans l'aristocratie ou le despotisme [...]. Ces derniers temps, les démocraties n'ont pas prospérés ${ }^{14} »$. La tendance consistant à faire barrage à la démocratie et à attribuer sa récente propagation uniquement aux «erreurs" des responsables persista, malgré l'influence de Rotteck, au niveau intermédiaire des dictionnaires conversationnels. L'Encyclopédie d'aujourd'hui (1838) de 
Brockhaus concédait certes que «la démocratie, en tant que véritable domination du peuple» avait trouvé "son terrain en Amérique». À la différence de Tocqueville, l'auteur considérait cependant que, même dans ce pays, la démocratie n'était pas nécessairement définitive. S'agissant de l'Europe, il considérait que "jamais elle n'y apparaitrait ni ne s'enracinerait ", si ce n'est à la suite d'une défaillance politique. La démocratie n'est selon lui effectivement possible que dans des conditions imaginées comme extrêmes (diffusion générale de la culture supérieure ou conditions primitives). La tendance démocratique moderne, qui faisait passer au premier plan de médiocres " intérêts matériels ", était considérée comme funeste ; on préconisait de la freiner. La démocratie était donc non seulement rejetée en tant que forme de domination ou de gouvernement, sur la base d'arguments traditionnels, mais aussi récusée comme dégradante, au nom d'«intérêts intellectuels ou moraux supérieurs ${ }^{315}$ ». On peut supposer que la vision développée dans l'encyclopédie Brockhaus était représentative de la majorité du public cultivé du Vormärz. Cette tendance très répandue consistant à reléguer la "démocratie » à l'arrière-plan et à n'associer au concept rien de ce qui décrit sérieusement la réalité constitutionnelle, du présent ou même de l'avenir, est illustrée aussi par la remarque de Goethe selon laquelle « dans la jeunesse, quand nous ne possédons rien ou que nous ne savons pas encore apprécier une tranquille possession, nous sommes démocrates", mais que "tous sans exception nous sommes aristocrates ${ }^{316}$ » à un âge plus avancé, en raison de la fortune acquise et de l'héritage à transmettre.

De même, Hegel n'inclut pas le principe démocratique comme un élément constitutif dans la théorie de l'État qu'il développe vers 1820 , dans la mesure où il n'appréhende pas ce principe, comme Görres, du point de vue de l'histoire des groupes statuaires [historisch-ständisch], mais dans la perspective égalitaire et individualiste de la Révolution française. Dans son "État concret», qui est « le tout articulé en ses cercles particuliers" et dont le membre atteint «sa destination effective et vivante à l'universel " "dans la sphère de sa corporation, de sa commune, etc.», [879] la démocratie n'a aucune place. L'idée que « tous doivent participer aux affaires de l'État » était pour lui insipide. «Cette représentation qui voudrait installer sans aucune forme rationnelle l'élément démocratique dans l'organisme de l'État, lequel n'est tel que grâce à une telle forme» renvoie selon Hegel à une «pensée superficielle». À «la détermination abstraite d'être membre de l'État ", Hegel oppose l'appartenance à un ordre [Stand] ou à une corporation, le principe d'une représentation ne pouvant faire sens que sur la base d'une telle appartenance, à l'inverse de la représentation des « individus-singuliers », de la "multitude ». Contre une vision atomistique et abstraite, Hegel formule la conception - expressément non démocratique - d'un État conçu comme "une organisation de maillons tels qu'ils sont pour soi des cercles» : en lui, " aucun moment ne doit se montrer comme une multitude inorganique ». Il faut rejeter « la représentation qui, là où elles accèdent au politique, c'est-à-dire au point de vue de la plus haute universalité concrète, dissout de nouveau en une multitude d'individus les communautés déjà présentes-là ${ }^{317} »$. Hegel a conçu en pensée la constitution de son "État concret » comme un organisme ordonné sur une base corporative et gouverné par des fonctionnaires sensés et cultivés, en se passant des concepts traditionnels de " démocratie » et d'" aristocratie ». Il les rejette lors même qu'elles sont "rabaissées au rang de moments dans la monarchie constitutionnelle». "De telles différences simplement quantitatives » n'apportent rien au «concept de la Chose». Il n'est selon lui pas pertinent non plus «que l'on ait, à l'époque présente, tant parlé de l'élément 
démocratique ou aristocratique dans la monarchie ; car les déterminations que l'on a en vue à ce propos, dans la mesure précise où elles ont lieu dans la monarchie, ne sont plus quelque chose de démocratique ou d'aristocratique ${ }^{318} »$. Pour Hegel, l'adaptation de la théorie traditionnelle de la constitution mixte à la constitution moderne était ainsi dénuée de sens. L'aristocratie et la démocratie, en tant que formes de domination anciennes ou en tant qu'éléments ou principes, ne présentaient aucune valeur de référence pour l'État de fonctionnaires monarchique et corporatif, conçu comme l'expression de la rationalité politique la plus élevée. Sur le plan conceptuel, la démocratie est restée en dehors d'un tel droit constitutionnel allemand, mais hors d'Allemagne, elle a joué selon Hegel un rôle considérable. Il décrit ainsi «la constitution française de l'an III sous Robespierre ", comme " démocratique », car elle fut pour une large part une démocratie directe. Hegel note qu'elle fut «adoptée par tout le peuple, mais bien sûr d'autant moins mise en œuvre ${ }^{319}$ ». On pouvait affirmer qu'elle était, selon lui, «quelque chose de fabriqué », ne reposant que sur un "pur et simple amas atomistique d'individus ${ }^{320} »$. Pour Hegel, il était entendu qu'un tel extrême ne pouvait se réaliser sur la durée, tout comme il était clair que la démocratie jacobine faisait peser en permanence une menace sur l'ordre rationnel. Hegel avait ainsi localisé la démocratie dans le contexte de la Révolution. Elle avait donc bien reçu sa place nécessaire dans le progrès de l'autoréalisation de l'esprit de liberté. Elle avait cependant introduit non une réconciliation, mais une nouvelle désunion et était restée historiquement en retrait du stade le plus élevé atteint par l'État rationnel au sens de la philosophie du droit de Hegel. La démocratie, en tant que "souveraineté du peuple ", [880] d'après des "pensées confuses au fondement desquelles réside la représentation inculte du peuple", qui font du peuple "une masse informe qui n'est plus aucun État ${ }^{321}$ ", constituait déjà pour Hegel, bien qu'elle constituât un danger actuel, un stade dépassé, une phase qu'avait surmonté le processus historique, lequel, avant tout chez les peuples germaniques, avait progressé, par-delà la démocratie jacobine, vers une nouvelle réconciliation et une nouvelle liberté. C'est en cela que réside l'efficacité politique de la conception hégélienne : la démocratie était privée du pouvoir suggestif associé au progrès et à ce qui est incontournable; elle était déjà "derrière ", supplantée par une idée supérieure de l'État, qui n'avait pas besoin de se montrer réactionnaire ou d'incarner défensivement une forme de restauration vis-à-vis de la démocratie. Directement ou indirectement, cette dépréciation de la démocratie dans la philosophie de l'histoire resta vivante en Allemagne jusqu'à la conception d'une «idée de l'État» proprement allemande, élaboration conceptuelle qui atteignit son paroxysme durant la Première Guerre mondiale, en opposition à l'Europe occidentale.

\section{La situation vers 1848}

11 À tout cela s'opposaient, il est vrai, jusqu'à 1848, plus particulièrement en Allemagne du Sud, les effets résultant de l'introduction, par Rotteck, de la notion de démocratie dans le droit constitutionnel ${ }^{322}$. Il convient également de mentionner Zachariä, spécialiste du droit constitutionnel à Heidelberg, lequel voulait laisser agir dans la monarchie constitutionnelle deux partis - un parti « royaliste » et un parti « démocratique » - et faire en sorte que la formation du gouvernement dépendît du plus fort de ces deux partis au Parlement ${ }^{323}$. Cette tendance démocratique et parlementaire faisait écho au concept de "monarchie démocratique », qui était bien connu en Allemagne, mais peu utilisé, et qui ne parvint finalement pas à acquérir une grande importance, même après 
sa propagation en 1848, la ligne définie par Rotteck et Zachariä ne se confirmant pas suffisamment dans la réalitée ${ }^{324}$. La thèse selon laquelle "l'Europe monarchique » devait elle aussi chercher, "non pas à réprimer, mais à prendre en compte et à organiser correctement les éléments démocratiques ${ }^{325}$ " (Bluntschli 1857) se maintint encore longtemps. En 1860, Biedermann apporta une nuance supplémentaire, en plaçant ses espoirs dans « la meilleure partie de la démocratie en Allemagne ", à savoir celle qui a acquis la conviction que « même au sein d'une forme constitutionnelle monarchique, la satisfaction des aspirations démocratiques raisonnables à la liberté politique est tout à fait possible, comme le prouve l'exemple de ce qu'on appelle en Belgique la monarchie démocratique ${ }^{326}$ ». Biedermann évoquait cette "meilleure partie de la démocratie", basée sur l'expérience que la démocratie ou les démocrates, dans leur simple participation à l'État et à la société - les " éléments démocratiques »-, [881] n'avaient justement pas recherché la voie de la médiation au moyen du compromis que représentait la monarchie constitutionnelle. Contrairement à la logique de donnantdonnant évoquée précédemment, le libéralisme et la démocratie s'étaient éloignés l'un de l'autre en Allemagne, comme l'avait montré la révolution de 1848. Dans la théorie du constitutionnalisme libéral, telle que la développa Dahlmann dans sa Politique, la notion de «démocratie » fut écartée. Dahlmann évitait le terme, bien qu'il se prononçât expressément en faveur du principe de représentativité et contre le principe d'assemblées territoriales fondées sur des états ${ }^{327}$. Cependant, les réticences de Dahlmann envers la démocratie, le mot et la chose, représentaient la ligne la plus «à droite » du libéralisme vers le milieu du siècle. À l'autre extrémité se trouvait Gervinus, pour qui l'histoire, depuis la fin du Moyen Âge, n'était qu'« une lutte entre les idées démocratiques, jetées parmi les hommes par la Réforme, et les institutions aristocratiques médiévales ». Il définissait ce qui est démocratique au moyen de valeurs typiquement libérales, comme "l'esprit de l'individualisme", "la liberté ", "l'éducation », "une plus grande liberté dans les croyances religieuses et le savoir, dans les droits politiques, la propriété et les affaires commerciales ${ }^{328} »$. Gervinus voyait le mouvement démocratique progresser irrésistiblement. Si cette seule conviction le relie déjà à Tocqueville, il était également proche de la conception de la démocratie proposée par ce dernier, dans la mesure où il concevait la démocratie à venir sous l'angle du nivellement de la "société », de l'«aspiration à l'égalité de toutes les conditions ", de la volonté d'émancipation des masses et des "effets produits par la littérature [...], qui se démocratise également dans toutes les parties de l'Europe ${ }^{329} »$. Comme Tocqueville, Gervinus voyait la démocratie en cours de réalisation atteindre son apogée en Amérique du Nord. Sans être lui-même un démocrate radical, il était conscient des progrès nécessaires pour parvenir à une démocratie égalitaire ; il chercha à fonder cette tendance de manière libérale, afin de voir les idéaux libéraux se réaliser pour l'avenir. Gervinus n'était pas le seul à considérer ainsi les États-Unis. Robert von Mohl, Karl Salomo Zachariä et Friedrich Murhard avaient déjà éprouvé auparavant la pertinence de leur concept de démocratie au contact du modèle américain et y avaient trouvé une confirmation de l'hypothèse qu'un gouvernement populaire [Volksherrschaft] représentatif (Mohl 1835) est possible ${ }^{330}$.

12 Tous ces savants allemands connaissaient De la démocratie en Amérique (1835-1840) de Tocqueville. Cependant, aucun d'eux n'avait pleinement accepté les présupposés et les implications de sa réflexion. Mohl, en particulier, l'admirait certainement, l'avait étudié en profondeur (y compris par la suite), mais s'opposait à lui sur le point central (l'égalité) et rejetait la démocratie égalitaire, qui allait au-delà d'une simple 
participation dans l'État et la société des États monarchiques parlementaires (l'« élément démocratique ») ${ }^{331}$. [882] Aux yeux de Mohl, en effet, la démocratie conduisait nécessairement à la « médiocrité », sur le plan du personnel politique et des institutions, ainsi que sur le plan intellectuel. Ni l'Amérique du Nord ni la Suisse ne constituaient selon lui des modèles souhaitables, ni même possibles pour les monarchies européennes. Mohl, qui représente ici de manière emblématique la vision qui prédomine dans le libéralisme allemand, appréciait probablement les analyses et les jugements subtils de Tocqueville, en particulier lorsque qu'ils mettent en évidence les dangers de la démocratie égalitaire, mais il ne pouvait suivre Tocqueville sur le fond, c'est-à-dire dans ses prévisions. En 1835, Tocqueville utilisait le modèle nord-américain pour définir la "démocratie» comme un principe historique universel du monde moderne, qui - selon un processus apparu d'abord en Amérique, mais dont l'amorce était également visible en Europe - s'était détourné du mode de vie aristocratique et se caractérisait selon lui par la marche vers «l'égalité des conditions». Tocqueville pensait que tout dérivait de ce principe dans la démocratie américaine. L'Europe lui semblait s'orienter dans la même direction. "Une grande révolution démocratique s'opère parmi nous. » Cette révolution n'est ni accidentelle ni réversible. Elle constitue davantage « un fait providentiel »: «Il est universel, il est durable, il échappe chaque jour à la puissance humaine; tous les événements, comme tous les hommes, servent à son développement ${ }^{332}$.» La conception tocquevillienne de la démocratie est donc formulée à partir du principe de l'égalité des chances dans la société. Le phénomène d'un « état social [...] éminemment démocratique » et le " principe de la souveraineté du peuple ", en l'absence de séparation de l'État et de la société, vont de pair ${ }^{333}$. La liberté des citoyens est inhérente au concept de « démocratie », bien que le principe de majorité menace cette liberté $(1,2,7)$ et que la démocratie moderne puisse tout autant incliner vers la liberté que vers le despotisme ${ }^{334}$. Pour Tocqueville, le danger du despotisme démocratique, même s'il le redoutait lui-même, ne pouvait plus conduire à la reconstruction d'une société aristocratique, mais seulement à l'effort continu pour « faire sortir la liberté du sein de la société démocratique où Dieu nous fait vivre ${ }^{335}$ ». En d'autres termes, depuis la Révolution, la démocratie était devenue le destin inéluctable, prévu dans les plans divins, des nations européennes. On ne peut y échapper ni lutter contre elle ; on ne peut qu'échouer devant elle ou réaliser ce qu'elle a défini comme une tâche. Si l'esprit du monde de Hegel avait déjà laissé la démocratie derrière lui, la Providence de Tocqueville conduisait tout droit vers elle et en son sein. Cette compréhension du mouvement général et irrésistible vers la démocratie, fondée sur une lecture du cours de l'histoire universelle, s'inscrit explicitement dans l'héritage des Lumières et de la Révolution, sans toutefois se réduire à celui-ci : [883] le mouvement vers la démocratie était inévitable et devait par conséquent être accepté. Elle n'était toutefois plus considérée comme un progrès, mais davantage comme une fatalité.

13 La conception tocquevillienne de la démocratie n'eut pas un grand retentissement en Allemagne et resta globalement, au XIX ${ }^{\text {e }}$ siècle, sans effet sur le plan politique. Cette interprétation fondée sur une neutralité axiologique, doublée d'une acceptation du mouvement moderne de démocratisation, ne parvint pas à s'imposer entre la vision, idéologiquement marquée, d'un progrès vers la démocratie, d'un côté, et l'effroi qu'elle suscitait, de l'autre. Au moment même où Tocqueville devenait célèbre en Allemagne s'exprimaient dans le débat des hommes qui, contrairement à Tocqueville, étaient euxmêmes démocrates et faisaient de la démocratie une question d'engagement. Dans la lutte contre l'«aristocratie » ou contre le "parti réactionnaire» - et c'était bien ce 
dont il s'agissait forcément vers 1840 -, la démocratie devint nécessairement une conviction relevant de la foi, cependant que les démocrates se constituaient en " parti ». L'interprétation de Tocqueville était dépassée lorsque qu'était, par exemple, affirmé non sans pathos que la "démocratie " ne constituait pas seulement "une opposition contre les gouvernants ou un changement constitutionnel ou politicoéconomique particulier ", mais " annonçait aussi une totale transformation de l'état de ce monde et une vie sans précédent dans l'histoire, originairement nouvelle ». Il fallait comprendre que "la démocratie était une religion». Le parti démocratique était encore faible et négatif pour l'heure, mais c'était le début d'un chemin vers le but : « un monde jeune et glorieux dans lequel toutes les dissonances actuelles s'aboliront dans une unité harmonieuse "; car, "par ses principes mêmes", le parti démocratique représente "le général, l'universel ${ }^{336}$ ». Ce pathos et cette prétention à l'absolu dépassent la tendance libérale consistant à vouloir harmoniser des " principes » ou des "éléments» - dont l'élément "démocratique». L'idée que les "démocrates" se faisaient d'eux-mêmes les conduisit à se détourner d'une doctrine libérale de l'État conciliante, mais aussi de la possibilité de penser et de s'efforcer d'instaurer la démocratie de manière pragmatique et, si besoin, dans un esprit de compromis. Ainsi, on mettait certes à nouveau en exergue le caractère contradictoire des partis démocratique et aristocratique de l'époque de la Révolution française, mais aussi, en parallèle, le fait que le « libéralisme bourgeois » ne se confondait pas avec elle. Comme le soulignaient des démocrates affichés - à l'instar de Wilhelm Marr (1844) -, le libéralisme bourgeois jouait probablement «le rôle principal en ces temps paresseux ", mais n'offrait à terme aucune perspective de succès. « Dans le cadre d'une révolution, la démocratie sans aucune limite revenait à jouer, face aux libéraux, le rôle des montagnards face aux girondins ${ }^{337}$. » Plus théorique, Fröbel inscrivit l'assurance d'une victoire de la démocratie dans une théorie des «stades de l'évolution culturelle»: l'État, peinant à s'affranchir de son intégration dans le domaine religieux, devait passer par les étapes d'une " république monarchique, aristocratique et démocratique ». Telles sont selon lui «les étapes principales sur le chemin culturel menant vers une société constituée ». Ce n'est que dans le cadre de la démocratie que "le règne de la moralité » se serait réellement constitué. Fröbel, parfaitement représentatif en cela des "démocrates " des années 1840 , fixe comme objectif la réalisation de la démocratie à l'échelle de l'État-nation pour le temps présent ou un proche avenir, sa réalisation universelle et transnationale pour un futur plus lointain. À la fin, on devait obtenir [884] « la fédération confraternelle de tous les hommes », organisée démocratiquement, "le gouvernement général du genre humain par lui-même », en marche vers l'étape d'une « fédération générale de tous les États ${ }^{338}$ ".

Vers 1840, semble-t-il, la tendance consistant à opposer la "démocratie » non seulement aux constitutions allemandes existantes, mais aussi à leur évolution parlementaire et libérale, au sens des idées de Rotteck, et à l'assimiler à la " république " s'accentua. L'encyclopédie Brockhaus (1840) rattache la « démocratie » à "ce que l'on entend ces derniers temps sous le mot de "république" " et ajoute, dans l'article "République ", qu'il serait impossible de réaliser durablement la démocratie ou la république ${ }^{339}$. Seuls les " démocrates » ou les « républicains » entendaient rendre possible l'impossible. Au début des années 1840, ils étaient désignés - et se désignaient généralement eux-mêmes - comme des "radicaux». Ce mot, depuis longtemps en usage en Angleterre, s'était rapidement répandu dans le langage politique français après la loi sur la presse de 1835, qui proscrivit le terme de "républicains ». Les 
démocrates français, dans le contexte des événements de 1839, se divisèrent en " radicaux» et "modérés ${ }^{340}$ ». En Allemagne, le terme "radical» - déjà formé au $\mathrm{XVIII}^{\mathrm{e}}$ siècle ${ }^{341}$ - se diffusa rapidement à la même période, sous l'influence française, sans toutefois se rattacher aussi étroitement à un "parti» des démocrates. Tout comme le "radicalisme» avait exprimé en France l'opposition aux «modérés » (démocrates), il marqua très vite en Allemagne (à partir de 1842) la ligne de partage avec le libéralisme. En 1843, Arnold Ruge réclamait « la dissolution du libéralisme dans le démocratisme ». Cela signifiait selon lui reprendre le mouvement, interrompu par la Confédération germanique, des guerres de libération et des "démocrates radicaux " d'alors, « dont la formidable efficacité réside dans la régénération de la Prusse et le soulèvement du peuple tout entier contre Napoléon ${ }^{342}$ ».

Le «radicalisme» englobait la notion de démocratie, qui, même dans les écrits politiques, semble s'être effacée temporairement au début des années 1840. Au cours des années 1840 , la "démocratie » devint de plus en plus concrète en termes de contenu. Elle recouvrait la souveraineté populaire, l'unité nationale et les liens entre les peuples, l'égalité politique (mais aussi sociale, de plus en plus), avec des conséquences sur les revendications en matière de droit de vote et autres « droits du peuple ». L'ensemble de ces attributs de la démocratie étaient également inclus dans le concept de " radical $^{343}$ ». Mais le terme de «radical » renvoyait en plus, en Allemagne, vers 1840 , à un mode de pensée marqué sur le plan philosophique, notamment par l'hégélianisme (de gauche), et qui, dans son inclination pour les théories extrémistes au sens moderne, volontariste, du terme, penchait non seulement vers la démocratie (= la république), [885] mais aussi vers l'intensification de celle-ci dans le socialisme ou le communisme. Ainsi, le terme de « radical » pouvait effectivement aller de pair avec le terme «démocratique » - y compris dans l'expression « radical-démocrate ${ }^{344} »-$; il pouvait aussi s'en éloigner derechef, lorsqu'il était compris en un sens si extrême qu'il plaçait la démocratie citoyenne, fût-elle égalitaire et républicaine, en deçà de projets plus extrémistes pour l'avenir. Bruno Bauer (1847) suggérait ainsi, en citant Moses Hess (1843) : «En théorie, nous sommes les hommes les plus libres, les démocrates les plus purs, les communistes les plus radicaux - mais en théorie seulement, malheureusement ${ }^{345}$ ».

En 1848, le parti des démocrates, qui avait déjà existé sous la forme d'une communauté de sensibilité dans la phase pré-parlementaire, apparut dans les associations populaires et dans les parlements du Reich et des différents États [allemands]. Il se démarquait ainsi - sans toutefois se différencier nettement de ces deux groupes - des radicaux (les communistes) ${ }^{346}$, d'une part, et des libéraux (les partisans de la monarchie constitutionnelle), d'autre part. Si les démocrates, dans leurs associations et lors de leurs deux congrès nationaux, cherchèrent fortement à se rassembler à la gauche du libéralisme, sous le slogan de la "république démocratique ", et à organiser leur unité en vain -, ils ne parvinrent pas à définir clairement la démocratie à laquelle ils aspiraient. À côté de l'engagement en faveur de la République, des remarques dépréciatives sur la «faiblesse du libéralisme » et l'affirmation d'une polarisation du peuple entre le "parti démocratique », d'une part, et le parti "conservateur " ou " constitutionnel ${ }^{347}$ ", d'autre part, il y avait des formules conciliantes, comme dans le programme proposé par l'Association patriotique (=démocratique) principale [Vaterländischer Hauptverein] en juillet 1848, à Stuttgart: "Notre programme ne se réalisera que dans le cadre de la démocratie, sous la forme d'une monarchie 
constitutionnelle fondée sur une base démocratique ou sous la forme de la république ${ }^{348}$. »

17 Si la frontière avec le libéralisme restait fluctuante, la Révolution avait accéléré et accentué le divorce entre celui-ci et la démocratie. Pendant et après la Révolution, les démocrates étaient par conséquent considérés comme des "ennemis de l'ordre ". L'ostracisation de la démocratie demeurait un aspect typique de la mentalité politique en Allemagne depuis 1848/49. Le slogan de 1848/49 - « contre les démocrates, seule la troupe est bonne ${ }^{349}$ » - correspondait à l'expérience de cette année-là et il resta vivant dans la mémoire. Les "soldats" avaient été associés au succès, la "démocratie » au soulèvement contraire à l'ordre et à l'échec. Cette tendance se confirma une nouvelle fois en 1866 aux yeux des Allemands, lorsque les démocrates, favorables à la solution grande-allemande, furent défaits en même temps que l'Autriche et les États intermédiaires $^{*}$ [Mittelstaaten]. [886] Par la suite, la création de la Confédération de l'Allemagne du Nord et de l'Empire scella le clivage, déjà établi en 1848 par Simon, entre les « conservateurs» et les « constitutionnalistes » (en fin de compte victorieux), d'une part, et les « démocrates », d'autre part.

La rupture avec le libéralisme - dans la mesure où il fallait entendre par là, depuis 1867, les nationaux-libéraux - était désormais définitive. De surcroît, au sein même de la démocratie, une autre divergence était apparue, qui s'était également annoncée dès les années 1840: la scission entre une démocratie sociale (des travailleurs) et une démocratie (bourgeoise), qui pouvait se définir comme libérale (de gauche) ou comme républicaine.

\section{La « démocratie sociale »}

Dans l'espace germanophone, la « démocratie sociale » s'est d'abord développée sur le plan théorique dans les associations de compagnons ouvriers en France et en Suisse à partir de 1834, dans la mesure où les intellectuels et les compagnons ouvriers qui se sentaient démocrates, à commencer par Weitling, revendiquaient non seulement leur adhésion à la république, mais déduisaient également du principe d'égalité d'amples implications sur le plan social, jusqu'aux positions extrêmes que représentaient le " socialisme » ou, dans le cas de Weitling, le " communisme ». La Déclaration des droits de l'homme et du citoyen de 1793, les enseignements de Babeuf et de Buonarotti ont été reçus et assimilés. La république en tant que « domination du peuple » et produit de la "révolution sociale ${ }^{350}$ » était comprise dans une perspective d'unité nationale et de fédéralisme international, mais aussi et surtout comme une forme constitutionnelle offrant la possibilité de réaliser une société nouvelle. En termes de contenu, il s'agissait donc d'une "démocratie sociale", par opposition à une démocratie purement "politique ", même si le terme était encore peu utilisé ou du moins n'était pas encore largement répandu ${ }^{351}$. Les associations de travailleurs allemandes ont ainsi pris part à un mouvement qui a débuté après le milieu des années 1830 en Belgique et en France. Dès 1836-1837, Le Débat social - Journal démocrate socialiste parut à Bruxelles ${ }^{352}$. En France, vers 1840 , les « radicaux $^{353}$ » (Ledru-Rollin), sous l'influence de Louis Blanc, Leroux et d'autres, se sont orientés vers un programme résolument " social », de sorte, que parmi les "démocrates radicaux », les expressions "démocrates sociaux » ou "démocrates socialistes " étaient désormais également possibles, bien que, semble-t-il, elles ne fussent devenues plus courantes qu'à partir du milieu des années $1840^{354}$. 
Lorenz von Stein n'utilisait pas encore le terme de "démocratie sociale » dans son premier ouvrage (1842), où il opposait déjà, cependant, la révolution " politique » et la révolution "sociale", ouvrant ainsi la voie à une interprétation sociologique de la question de la constitution de l'État. Sur la base de cette distinction, Friedrich Engels prédit en 1844, s'agissant de l'Angleterre, une lutte des pauvres « contre les riches (la classe moyenne) », [887] « de la démocratie contre l'aristocratie ». La démocratie vers laquelle s'orientait l'Angleterre serait selon lui une « démocratie sociale ${ }^{355}$ ». À la faveur d'un déplacement de l'accent vers le mouvement social-révolutionnaire, on a également pu parler de socialisme "démocratique ${ }^{356}$ ", pour reprendre la formule utilisée par Karl Grün en 1845. Lors de la révolution de 1848, notamment au sein des associations de la Fraternité des travailleurs, les expressions « démocrates sociaux » ou "sociaux-démocrates" devinrent courantes, afin de distinguer ces groupes des démocrates - au sens strictement politique du terme - présents dans les associations pour la démocratie ou au sein des parlements. C'est par exemple dans cette perspective que Friedrich Hecker utilise ces expressions dans le discours tenu le 5 mars 1848 dans la cour du château de Heidelberg. Stefan Born écrit ainsi en 1849, au sujet de la Fraternité : «Nous nous sommes déclarés ouvertement [...] sociaux-démocrates ${ }^{357}$.» Durant la révolution, on parla également, à plusieurs reprises, de république " démocratique sociale » ou « sociale-démocrate ${ }^{358}$ ".

21 Après la révolution, les termes de «démocratie sociale » ou de "social-démocratie " ont été précisés sur le plan conceptuel par Lorenz von Stein. Dans sa théorie, développée à partir de l'exemple français, la démocratie "sociale» se situe au croisement du «mouvement social» et du «mouvement purement politique». Le premier vise à donner le pouvoir [Herrschaft] à la "classe des non-possédants", jusqu'alors dominée; le second, qui renvoie au "mouvement républicain ou démocratique", vise une "constitution libre", fondée sur le principe de l'égalité civique $^{359}$. Si la tendance sociale et la tendance constitutionnelle se rejoignaient, la première se réalisait dans l'administration, la seconde dans la constitution de l'État. La combinaison des deux mouvements était conçue comme la «démocratie sociale ». Le principe «de la démocratie sociale renvoie donc au suffrage universel pour ce qui concerne la constitution et à l'abolition de la dépendance sociale de la classe laborieuse pour ce qui concerne l'administration. Dans la démocratie sociale, la constitution est donc la composante démocratique, l'administration la composante sociale ». Au-delà des différences qui tiennent au caractère dialectique de sa pensée, dans la lignée de Hegel, Stein n'est pas ici sans évoquer Tocqueville ${ }^{360}$, en ce qu'il établit comme ce dernier la nécessité de cette évolution, mais envisage également pour l'avenir une alternative déjà présente en elle - la "réforme» ou la "révolution" sociale. En espérant conjurer la révolution à travers la réforme, il anticipait la venue de l'État social ${ }^{361}$. [888] La démocratie telle qu'elle s'est développée jusqu'à présent, dans la mesure où «elle fait de la constitution l'enjeu principal", s'avère selon lui "impuissante». "Si elle se concentre sur l'administration, elle n'est plus une démocratie. La fin de cette démocratie intervient dès que les deux classes s'accordent sur leur véritable intérêt mutuel [...]. La transition de la démocratie vers cette nouvelle forme s'esquisse déjà dans le mot d'ordre de la démocratie sociale ${ }^{362}$."

Dans les années 1860, l'expression "démocratie sociale » en tant qu'auto-désignation est finalement devenue une notion courante, évoquant le lien entre le mouvement ouvrier et la démocratie, par opposition à une démocratie purement " politique » ou 
«bourgeoise ». Plus nettement qu'en 1848-1850, l'expression tendit ainsi à désigner un parti. Alors que Lassalle lui-même était encore réservé sur ce point, Jean Baptist von Schweitzer parlait ainsi, dès le 13 octobre 1863, de «la lutte du parti social-démocrate » contre «le parti libéral bourgeois»; le 15 décembre 1864, le premier numéro du journal de l'Association générale des travailleurs allemands [Allgemeiner Deutscher Arbeiterverein] paraissait sous le titre Le Social-démocrate [Der Social-Demokrat] ${ }^{363}$. En 1867, on lisait dans Le Précurseur [Der Vorbote] de J. P. Becker :

«Alors que la démocratie exclusivement politique entend rouvrir pleinement la révolution prise dans une impasse et la parachever dans la république bourgeoise, la social-démocratie veut contribuer à accélérer cet achèvement, afin de le dépasser immédiatement et d'inaugurer la fondation d'un nouvel État populaire et d'une nouvelle époque culturelle, l'époque sociale ${ }^{364}$. »

Ce constat se rattachait à l'idée que la "démocratie politique " s'était hissée "au niveau du concept plus cohérent, plus avancé et plus élevé de social-démocratie ${ }^{365}$ ». Lorsque le Parti ouvrier social-démocrate [Sozialdemokratische Arbeiterpartei] se dota d'un programme à Eisenach en 1869, la "social-démocratie » devint le nom, établi durablement, du futur SPD [Sozialdemokratische Partei Deutschlands].

Si, dans l'histoire du concept de «social-démocratie » entre 1840 et 1870, l'objectif social était encore lié au modèle constitutionnel, traditionnel pour la " démocratie ", dans le langage politique, une étape supplémentaire a été franchie vers le milieu du siècle, lorsque le terme de " démocratie » fut appliqué uniquement à la société. Depuis les années 1840, une société égalitaire débarrassée de l'«aristocratie " pouvait être décrite comme " démocratique ", voire comme "socialiste ", et la société en question pouvait par conséquent être envisagée comme étant engagée dans un processus de démocratisation.

«La société est de plus en plus équilibrée, se démocratise ; la prétention des classes [Stände] supérieures à jouir du privilège de l'éducation, des plaisirs et du pouvoir politique [politische Herrschaft], à donner le ton dans le domaine des mœurs publiques, ne cesse d'être rabaissée, tandis que les revendications des classes inférieures s'élèvent de plus en plus ${ }^{366}$. "

Ludwig Häusser pouvait affirmer dans une perspective similaire que le code municipal de Bade de 1831 "a démocratisé les fondements de la société politique ${ }^{367}$ ». [889] À l'époque de la révolution de 1848-1849, la notion de "démocratisation de la société " était largement utilisée dans le langage politique et associée à l'idée qu'elle n'était pas nécessairement « liée à un régime politique donné » et qu'elle pouvait se manifester « au travers d'une certaine homogénéité du mode de vie, de l'habillement et de la société ${ }^{368}$ ». Cet usage des termes " démocratie » et " démocratisation », encore courant aujourd'hui, pouvait s'appliquer sans limites dans tous les domaines de la société. Il ne sera mentionné ici qu'à partir de sa racine, à savoir la séparation du social et du politique.

\section{Marx et Engels}

Marx et Engels adoptèrent une position sur le concept de démocratie que l'on ne saurait rattacher aux catégories de la démocratie libérale, radicale et sociale que nous avons développées jusqu'à présent ; ils entretenaient au départ des liens étroits avec l'ensemble de ces tendances, en particulier avec celles des démocrates radicaux et sociaux. À partir de 1844 , cependant, en se décrivant comme "communistes ", ils franchirent et dépassèrent clairement les stades de la démocratie mentionnés ci- 
dessus, sans toutefois cesser d'éprouver à l'égard de la démocratie - interprétée selon leur théorie - le sentiment d'un engagement ou d'une appartenance au sens large. Dans sa Critique de la philosophie du droit de Hegel, Karl Marx s'identifie explicitement à la démocratie associée à la phase républicaine de la Révolution française, que rejetait Hegel. À la souveraineté de l'État incarnée par le monarque, Marx oppose la souveraineté du peuple, qui seule permet non seulement de placer une «forme», la " constitution politique ", au-dessus du peuple, mais aussi de réunir "le contenu et la forme ». « Dans la monarchie, le tout, le peuple, est subsumé sous l'une de ses manières d'être, la constitution politique; dans la démocratie la constitution elle-même n'apparaît que comme une détermination, à savoir comme autodétermination du peuple. » La démocratie serait ici «l'essence de toute constitution étatique, l'homme socialisé en tant qu'une constitution politique particulière ", qui se distingue de toutes les autres constitutions par le fait qu'en elle, une "existence humaine » est rendue possible, "tandis que dans les autres, l'homme s'apparente à l'existence légale ", "l'homme politique " étant pour cette raison séparé de "l'homme non politique, privé ». La " démocratie », ainsi conçue, ne pouvait être qu'une république ; mais celleci était alors plus "qu'une simple constitution politique». Selon lui, dans cette république du futur, la démocratie de la liberté de l'homme (non divisé en lui-même) se réalisera. Pour Marx, les stades précédents étaient les suivants : 1 . la " démocratie de la non-liberté » au Moyen Âge, où « vie du peuple et vie de l'État sont identiques »; 2. la séparation de l'État et de la société («abstraction de l'État, abstraction de la vie privée ») à l'époque moderne. La démocratie était donc conçue par Marx comme l'autoréalisation politique de l'être humain, à travers un processus en trois étapes faisant se succéder "l'aliénation » dans le «dualisme réel » (Moyen Âge), la scission dans le "dualisme abstrait " (l'époque moderne) et la réconciliation dans l'avenir de «l'homme socialisés ${ }^{369}$ ».

[890] La démocratie remplaçait donc l'État hégélien. Elle était passée du stade préliminaire (Hegel) au stade final d'un processus historique libéré de la tutelle divine. Peu après, la révolution prolétarienne et le communisme furent ajoutés à cette approche pour désigner le but de l'histoire et placés au service de sa concrétisation.

Chez Friedrich Engels, qui dès 1843 avait désigné la Révolution française comme "l'origine de la démocratie en Europe », cette approche s'articula à l'idée que « la vraie liberté et la vraie égalité, c'est-à-dire le communisme » devaient se développer à partir de la démocratie apparente et fausse ${ }^{370}$. Il précisait deux ans plus tard : « La démocratie, c'est aujourd'hui le communisme. [...] La démocratie est devenue principe prolétarien, principe des $\operatorname{masses}^{371}$. " L'assimilation du «communisme » à la "démocratie », selon l'approche initiale et explicitement pré-communiste de Marx, n'était valable que pour la phase finale à venir et dans la croyance à laquelle devait adhérer le "parti » des communistes. Pour l'instant, cette équivalence était revendiquée à titre de programme, mais n'était pas réalisée dans cette démocratie dont se réclamait, avant et pendant la révolution, le "parti des démocrates ». Il ne pouvait y avoir d'alliance politique avec celui-ci que pour un temps limité et au moment opportun, comme en $1848^{372}$. C'étaient "les démocrates petits-bourgeois, [...] le parti de la petite bourgeoisie ", que l'on distinguait de la «bourgeoisie libérale », d'une part, et du "parti révolutionnaire des travailleurs ", d'autre part ${ }^{373}$. Dans la phase de la révolution libérale-démocratique et de son échec (1848-1850), les termes politiques «libéralisme", "démocratie " et « communisme " étaient entendus par Marx et Engels à la lumière de ceux qui les 
incarnaient dans la société, "bourgeois ", "petit-bourgeois » et "travailleurs " ("prolétariens »), et aussi, dans le passage, en un mouvement ascendant, de l'Étatnation monarchique fédéral à l'état-nation fédéral plus fortement centralisé puis à l'État-nation républicain et démocratique (communiste), unitaire et centralisée ${ }^{374}$. Sur un plan programmatique, cependant, la «démocratie" était déjà associée au "prolétariat ", à condition que la révolution ne soit pas menée au nom d'un objectif limité, "petit-bourgeois", et avortée avant son terme, mais qu'elle soit une «révolution permanente ${ }^{375}$ ». Ce programme intégrait évidemment la «socialdémocratie ", mais elle n'était pas conçue comme l'objectif final, dans la mesure où le "discours social-démocrate", dans ses compromis avec la démocratie petitebourgeoise, était trop peu révolutionnaire ${ }^{376}$. "Le prétendu parti social-démocrate » [891] que Marx, en 1852, évoquait rétrospectivement pour la France de 1848-1849, lui apparaissait comme une « nouvelle Montagne » avec des accents sociaux. « Le caractère propre de la social-démocratie se résumait à ceci qu'elle réclamait des institutions républicaines démocratiques comme moyen, non pas de supprimer les deux extrêmes, le capital et le salariat, mais d'atténuer leur antagonisme et de le transformer en harmonie ${ }^{377}$.» Le processus menant, à travers la «social-démocratie», de la "pure démocratie ${ }^{378}$ » au saut révolutionnaire (la "dictature du prolétariat»), qui devait ouvrir la voie à la démocratie véritable du communisme, fut pleinement développé sur le plan théorique par Marx et Engels entre 1843 et 1852. Tous les éléments développés ultérieurement n'apparaissent que comme de nouvelles applications ou des adaptations ${ }^{379}$.

Lassalle n'atteignit pas la précision théorique de Marx et Engels. Entre 1848 et 1863, il se trouva au cœur de la conception générale de la démocratie. Son importance ne réside pas dans son originalité sur le plan de l'histoire des concepts, mais dans la concrétisation politique des éléments d'ordre démocratique dans la situation comprise entre 1858 et 1864. Autrement dit, il prit acte de la séparation d'avec le libéralisme (le parti du progrès), qu'il opéra lui-même, et déclara que la "classe ouvrière " [Arbeiterstand] était porteuse de la démocratie. "La démocratie était le trait d'union entre la bourgeoisie et la classe ouvrière. En se débarrassant de ce nom et en le reniant, le camp de la bourgeoise a coupé ce trait d'union et l'on a planté l'étendard d'un mouvement bourgeois libéral, et non plus démocratique ${ }^{380}$. " Lassalle était proche de Marx en ce qu'il inscrivait lui aussi la "démocratie » - certes de manière plus vague encore - dans le mouvement du processus historique, même si, considéré du point de vue de Marx, il restait au stade de la "social-démocratie» et soulignait particulièrement fortement la relation, communément exprimée, entre démocratie et « nationalité » (« autodétermination ») $)^{381}$.

\section{L'éviction du concept de démocratie}

Dans les années 1860 et 1870, quand la fondation du Reich eut été préparée, conquise et accomplie, le "parti» des démocrates, dans la variété de ses formes et de ses organisations, s'inclina, et la victoire échut à ceux qui envisageaient la « démocratie » à partir de la tradition du « parti des aristocrates » ou du « libéralisme bourgeois ». Cette situation devint déterminante pour la conception dominante de la «démocratie » en Allemagne, [892] tant dans l'Empire allemand que dans les territoires allemands de la monarchie des Habsbourg. Si l'on tient compte du fait que depuis 1871, le concept de 
démocratie était resté vivant surtout dans le SPD et qu'il s'était développé dans une perspective "social-démocrate» ("État populaire») ou dans une perspective «marxiste », l'on comprend que le courant des démocrates seulement «bourgeois » se soit réduit et affaibli, de sorte que le mot « démocratie » n'a pas été utilisé dans les programmes des partis "libéraux» ou "progressistes » durant la période comprise entre 1878 et 1910. Seul le Parti populaire allemand [Deutsche Volkspartei], dont l'implantation se limitait au sud de l'Allemagne, y resta attaché. Il est ainsi révélateur que Friedrich Naumann, en 1910, ait caractérisé la réunion des partis à l'origine du nouveau Parti populaire progressiste [Fortschrittliche Volkspartei] comme une union des "démocrates du Sud» aux autres groupes libéraux ${ }^{382}$. Pour la vision conservatrice, l'affirmation formulée par Hermann Wagener dans le Dictionnaire de l'État [Staatslexikon] restait d'actualité, selon laquelle "la promesse fictive de la liberté et de l'égalité » occupait « une place centrale» au sein de la démocratie «pure» comme au sein de la démocratie "représentative ", la démocratie ne pouvant ainsi se réaliser solidement et de manière durable ${ }^{383}$. En pratique, les libéraux qui s'étaient tournés vers le nouveau Reich ne se démarquaient guère d'une telle conception. Heinrich von Sybel n'avait certes pas exclu la possibilité que le développement général de la constitution pût conduire à la démocratie dans un avenir lointain ${ }^{384}$, mais il n'autorisait pas de solutions démocratiques pour son époque et il soulignait - comme Treitschke - que la Selbstverwaltung* (prussienne), comme le self-government (anglais), était essentiellement non pas démocratique, mais aristocratique ${ }^{385}$.

Le concept de démocratie de Treitschke est le prototype de la fusion libéraleconservatrice au temps de la fondation du Reich et trace en outre la voie à suivre pour les élites cultivées attachées au Reich national, jusqu'après la Première Guerre mondiale. Revenant directement à Aristote, pour une part, et empruntant à Schleiermacher (voir supra) et à Roscher ${ }^{386}$, pour une autre part, Treitschke retourna radicalement l'idée d'un progrès historique vers la démocratie - telle qu'elle avait par exemple été développée par Gervinus et Fröbel -, en n'accordant pas à la démocratie la place d'honneur d'un stade pleinement développé, mais seulement le rang d'un stade préliminaire primitif sur la voie d'une " perfection ${ }^{387}$ " politique supérieure. Treitschke fixa ses critères à partir de l'objectif visé ou atteint d'une monarchie constitutionnelle allemande et prussienne fondée sur une unité politique aussi forte que possible combinée à une Selbstverwaltung largement aux mains des notables d'une "société civile» conçue, comme chez Aristote, comme fondée "par nature» sur l'inégalité. Selon lui, le principe de la démocratie est l'« égalité », qui contrevient à « l'organisation aristocratique de la société produite par la nature même des choses ", [893] de sorte que la démocratie n'est possible que si elle s'écarte de son propre principe et n'est donc plus cohérente. Treitschke montra ce phénomène non seulement pour la Suisse et les États-Unis d'Amérique, mais aussi pour le modèle classique, la démocratie athénienne, qui reposait sur une base esclavagiste et était en réalité la domination d'une « aristocratie de la masse » des citoyens de plein droit sur les esclaves ${ }^{388}$. La démocratie de l'égalité universelle contient une contradictio in adjecto; elle poursuit « un but qui est [...] inconcevable »; elle éprouve donc « le besoin compulsif de forcer un idéal en soi inaccessible ${ }^{389} »$. Treitschke n'accepte pas non plus l'association que l'on fait la plupart du temps entre liberté et démocratie, dans la mesure où il note que l'individualité était niée dans la démocratie ancienne et que la liberté est réalisée dans la monarchie moderne, bien qu'il refuse d'attribuer la liberté politique et personnelle à des formes d'État déterminées, comme le veut une «lubie » à la mode ${ }^{390}$. Si, pour Treitschke, la 
démocratie se révèle irréelle dans son principe et faiblement capable de s'adapter de manière pragmatique à la réalité, tant au regard d'une théorie politique "naturelle " (Aristote, Roscher) qu'à la lumière de l'expérience historique et de ses objectifs politiques, il décèle pourtant «une loi historique de démocratisation des formes de gouvernement » au sein des monarchies modernes, qui n'ont cependant fait qu'évoluer en conséquence, sans qu'il soit envisageable que la démocratie, en tant que forme étatique, prenne un jour le relais. Cette loi repose selon lui sur le fait que «la prospérité et l'éducation" se répandent de plus en plus et que «le droit de prendre une part active à la vie politique » s'ouvre ainsi «à des cercles de plus en plus larges». Cette tendance serait légitime "dans des limites raisonnables». En Allemagne, on a «malheureusement atteint la limite extrême - rien de plus déraisonnable ne peut être imaginé -, c'est-à-dire le droit de vote égal pour tous ${ }^{391} »$. Un tel dépassement de la limite contredisait la «nature» de la «société civile» et mettait en évidence la tendance au nivellement social et intellectuel, inhérente à toute démocratie selon Treitschke et qu'il n'a cessé de dénoncer. Au regard de critères d'évaluation " aristocratiques et bourgeois », la " nature de la démocratie » lui paraissait médiocre. Un séjour en Suisse (1864) conforta chez lui l'impression, qu'il avait anticipée, d'une « honorable médiocrité générale ${ }^{392}$ ». Il mettait celle-ci en relation avec la petite taille du pays et appliquait ainsi à son temps - c'est-à-dire, implicitement, à la question allemande - l'idée traditionnelle selon laquelle la démocratie n'est possible que dans des États de taille réduite et que seuls ces derniers peuvent demeurer démocratiques dans la durée. Une grande nation, importante à l'échelle de l'histoire mondiale (Hegel), ne pouvait et ne devait pas être constituée de manière démocratique.

L'expulsion de la démocratie hors de l'horizon politique qui était celui de la conscience officielle du Reich et de la nation allemande avait pour corrélat que la " démocratie » n'avait pas sa place dans le droit constitutionnel du Reich allemand. Pour Jellinek - se référant à la tradition depuis Aristote ou Machiavel -, la «démocratie " était incompatible avec la « monarchie ». [894] Par conséquent, dans le cadre d'une typologie différenciée, elle ne pouvait se présenter dans la réalité et n'être comprise sur le plan théorique que sous la forme d'une «république démocratique » (république $=$ «nonmonarchie $)^{393}$.

\section{La démocratie chrétienne}

31 Des analyses qui précèdent, on retiendra qu'après 1848 , il n'y avait plus en Allemagne de besoin durable d'insérer concrètement le concept de démocratie dans la pensée politique constitutionnelle - si ce n'est pour nier l'existant, c'est-à-dire en relation avec la révolution, dont les nouveaux extrêmes que représentaient le socialisme et le communisme s'emparaient de leur côté du concept de démocratie, le dépassaient et le rendaient superflu, ou se l'appropriaient sous la forme de la « social-démocratie ».

Si la «démocratie " était restée étrangère au type d'État allemand de la monarchie constitutionnelle, le terme n'avait guère été associé non plus à l'Église et au christianisme. En Allemagne, la communauté de vie de l'État et de l'Église se caractérisait par le fait qu'il existait bien un mouvement chrétien social, mais pas de mouvement démocrate-chrétien. Ce n'est que dans les années situées autour de 1848 que l'expression de "démocratie chrétienne" a joué un rôle, alors marginal. Si le mouvement «chrétien social» était acceptable sur le plan intraconstitutionnel, la 
tendance «démocrate-chrétienne " induisait au contraire une orientation révolutionnaire, qui était très éloignée des deux Églises, indépendamment des relations différentes qu'elles pouvaient entretenir avec l'État, et était combattue par les protagonistes chrétiens sociaux des deux confessions, comme Wichern et Ketteler ${ }^{394}$.

Ainsi, le terme de "démocratie chrétienne" était peu important pour les États allemands du XIXe siècle, même s'il n'était pas inconnu. En effet, il avait déjà été inventé en France en 1791, dans le cadre de la lutte contre l'Église, et s'était fortement répandu au cours des années 1830 et 1840 (Lammenais, Buchez), dans la mesure où il était resté particulièrement utile au regard du clivage entre la poursuite de la Révolution et le catholicisme renouvelée ${ }^{395}$. Ce besoin persista d'une nouvelle manière dans le contexte de la Troisième République, notamment après que Léon XIII, dès 1885, propagea l'idée d'une indifférence quant à la forme de l'État et rendit ainsi possible le «ralliement» des catholiques à la République française. Dans l'encyclique Graves de communi (1901), [895] les catholiques furent cependant enjoints de dégager leur "démocratie chrétienne » de toute implication politique et de la limiter, dans une forme de neutralité vis-à-vis des différentes formations étatiques, au domaine social et caritatif.

Au moment de la parution de l'encyclique, l'expression « démocratie chrétienne » était devenue courante dans d'autres pays que la France, notamment en Belgique et en Italie ${ }^{396}$. L'influence française, les encycliques de 1891 et de 1901 et le mouvement ouvrier chrétien naissant se traduisirent en Allemagne par l'apparition du terme de " démocratie chrétienne ", qui put être utilisé - non sans une certaine prudence quant à ses implications politiques - par les courants de "gauche » du catholicisme politique allemand. En règle générale, cependant, avant 1918, il était encore défini, en écho à l'encyclique de 1901, comme une tendance dangereuse («démochristianisme» en Italie) ${ }^{397}$. Par contre, pour le protestantisme, le terme demeura presque totalement étranger jusqu'en 1918. Et au-delà de cette césure, la "démocratie chrétienne» continua de renvoyer avant tout à la sphère catholique. En effet, l'idée de la démocratie chrétienne reposait essentiellement sur le présupposé que, dans les principaux pays où elle avait vu le jour, à savoir la France et l'Italie, un État-nation sécularisé avait été bâti dans ou sur un peuple presque exclusivement catholique, et que ce peuple, dans la mesure où il n'était pas devenu étranger à l'Église, devait accéder à un État démocratique moderne sans renoncer à son attachement aux traditions chrétiennes et catholiques.

\section{BIBLIOGRAPHIE}

Aretin, Johann Christoph von / Rotteck, Karl von (1838-1840) : Staatsrecht der constitutionellen Monarchie, 3 vol., $2^{e}$ éd., Leipzig ; éd. orig. Altenbourg, 1824-1828.

Aretin, Johann Christoph von / Rotteck, Karl von (1838) : Staatsrecht der constitutionellen Monarchie, vol. I, $2^{\mathrm{e}}$ éd., Leipzig. 
Arndt, Ernst Moritz (1908 [1814]) : « Über künftige ständische Verfassungen in Deutschland », in : Ausgewählte Werke, vol. XIII, Leipzig, p. 197-250.

Bachem, Karl (1930) : Vorgeschichte, Geschichte und Politik der Zentrumspartei, vol. VII, Cologne. Bachem, Karl (1931) : Vorgeschichte, Geschichte und Politik der Zentrumspartei, vol. VIII, Cologne. Bachem, Karl (1932) : Vorgeschichte, Geschichte und Politik der Zentrumspartei, vol. IX, Cologne. Balser, Frolinde (1965) : Sozial-Demokratie 1848/49-1863, $2^{\mathrm{e}}$ éd., Stuttgart.

Bauer, Bruno (1847) : Vollständige Geschichte der Parteikämpfe in Deutschland während der Jahre 1842-1846, 3 vol., Charlottenbourg.

Bluntschli, Johann Caspar / Brater, Karl Ludwig Theodor (1857) : Deutsches Staats-Wörterbuch, vol. II, Stuttgart.

Böckenförde, Ernst-Wolfgang (1963) : « Lorenz von Stein als Theoretiker der Bewegung von Staat und Gesellschaft zum Sozialstaat », in Alteuropa und die moderne Gesellschaft: Festschrift für Otto Brunner, Göttingen.

Boldt, Werner (1970) : Die württembergischen Volksvereine von 1848 bis 1852, Stuttgart.

Brockhaus (1818) : Conversations-Lexikon, 4 e éd., vol. II, Leipzig.

Brockhaus (1838) : Conversations-Lexikon der Gegenwart, vol. I, Leipzig.

Brockhaus (1840) : Conversations-Lexikon, $8^{\mathrm{e}}$ éd., Leipzig.

Brockhaus (1845) : Die Gegenwart. Eine encyklopädische Darstellung der neuesten Zeitgeschichte für alle Stände, vol. IX, Leipzig.

Bussmann, Walter (1952) : Treitschke, Göttingen.

Dahlmann, Friedrich Christoph (1835): Die Politik auf den Grund und das Maaß der gegebenen Zustände zurückgeführt, vol. I, Göttingen.

Duden (1963) : Etymologie. Herkunftswörterbuch der deutschen Sprache, éd. par Günther Drosdowski, Paul Grebe et al., Mannheim / Vienne / Zurich.

Eckermann, Johann Peter (1988) : Conversations de Goethe avec Eckermann, trad. J. Chuzeville, Paris.

Eckert, Hugo (1968) : Liberal- oder Sozialdemokratie. Frühgeschichte der Nürnberger Arbeiterbewegung, Stuttgart.

Elysard, Jules (1842) : « Die Reaction in Deutschland », Hallische und deutsche Jahrbücher für Wissenschaft und Kunst, 5.

Engels, Friedrich (1957 [1844]) : « Die Lage Englands. II. Die englische Konstitution », in : Marx, Karl / Engels Friedrich : Werke, vol. I, Berlin.

Engels, Friedrich (1959 [1845/46]) : « Das Fest der Nationen in London », in : Marx, Karl / Engels Friedrich : Werke, vol. II, Berlin.

Engels, Friedrich (1959 [1847]a) : « Die Kommunisten und Karl Heinzen », in : Marx, Karl / Engels Friedrich : Werke, vol. IV, Berlin.

Engels, Friedrich (1959 [1847]b) : « Grundsätze des Kommunismus », in : Marx, Karl / Engels Friedrich : Werke, vol. IV, Berlin.

Engels, Friedrich (1964 [1843]) : « Fortschritte der Sozialreform auf dem Kontinent », in : Marx, Karl / Engels Friedrich : Werke, vol. I, Berlin. 
Eschenburg, Theodor (1959) : «Tocquevilles Wirkung in Deutschland », in : Tocqueville, Alexis de : Werke und Briefe, éd. par J. P. Mayer, vol. I, Stuttgart.

Filthaut, Ephrem (1960) : Deutsche Katholikentage 1848-1958 und soziale Frage, Essen.

Fogarty, Michael (1959) : Christliche Demokratie in Westeuropa 1820-1953, Bâle / Fribourg / Vienne.

Franz, Eckhart G. (1958) : Das Amerikabild der deutschen Revolution von 1848/49, Heidelberg.

Fröbel, Julius (1847) : System der socialen Politik, vol. II, Mannheim.

Gentz, Friedrich (1844 [1819]) : « Über den Unterschied zwischen den landständischen und Repräsentativ-Verfassungen. Beilage zu den Carlsbader Protokollen », in : Klüber, Johann Ludwig / Welcker, Carl : Wichtige Urkunden für den Rechtszustand der deutschen Nation, mit eigenhändigen Anmerkungen von Johann Ludwig Klüber, Mannheim.

Gerhardt, Martin (1931) : Johann Hinrich Wichern. Ein Lebensbild, Hambourg.

Gervinus, Georg Gottfried (1853) : Einleitung in die Geschichte des neunzehnten Jahrhunderts, Leipzig.

Görres, Joseph (1929 [1819]) : «Deutschland und die Revolution », in : Gesammelte Schriften, vol. XIII, Cologne.

Grün, Karl (1845) : Die soziale Bewegung in Frankreich und Belgien, Darmstadt.

Guizot, François (1849) : De la démocratie en France, Paris.

Häusser, Ludwig (1851) : Denkwürdigkeiten zur Geschichte der Badischen Revolution, Heidelberg.

Hansemann, David (1834) : Preußen und Frankreich, $2^{\mathrm{e}}$ éd., Leipzig.

Hegel, Georg Wilhelm Friedrich (1930 [1831]) : « Über die englische Reformbill », in : Sämtliche Werke, vol. XX, Stuttgart.

Hegel, Georg Wilhelm Friedrich (2013) : Principes de la philosophie du droit, trad. J.-F. Kervégan, Paris.

Hess, Moses (1961) : Philosophische und sozialistische Schriften 1837-1850, éd. par Auguste Cornu et Wolfgang Mönke, Berlin.

Humboldt, Wilhelm von (1936) : Gesammelte Schriften, vol. XVII, Berlin / Leipzig.

Jellinek, Georg (1960 [1900]) : Allgemeine Staatslehre, $7^{\mathrm{e}}$ réimp. de la $3^{\mathrm{e}}$ éd., Bad Homburg.

Ketteler, Wilhelm Emanuel von (1924) : Schriften, éd. par Johannes Mumbauer, $2^{\mathrm{e}}$ éd., Munich.

Klüber, Johann Ludwig / Welcker, Carl (1844) : Wichtige Urkunden für den Rechtszustand der deutschen Nation, mit eigenhändigen Anmerkungen von Johann Ludwig Klüber, Mannheim.

Lassalle, Ferdinand (1919a) : Der italienische Krieg und die Aufgabe Preußens, in : Gesammelte Reden und Schriften, vol. I, Berlin.

Lassalle, Ferdinand (1919b) : « Rede von 19.5.1863 », in : Gesammelte Reden und Schriften, vol. III, Berlin.

Maier, Hans (1964) : « Herkunft und Grundlagen der christlichen Demokratie », in : Hürten, Heinz (éd.) : Christliche Parteien in Europa, Osnabrück.

Maier, Hans (1965) : Revolution und Kirche. Studien zur Frühgeschichte der christlichen Demokratie 1798-1901, $2^{\mathrm{e}}$ éd., Fribourg.

Marr, Wilhelm (1846) : Das junge Deutschland in der Schweiz, Leipzig. 
Marx, Karl (1962 [1875]) : «Randglossen zum Programm der deutschen Arbeiterpartei », in : Marx, Karl / Engels Friedrich : Werke, vol. XIX, Berlin.

Marx, Karl (1975) : Critique du droit politique hégélien, trad. A. Baraquin, Paris.

Marx, Karl (1964) : Le 18 Brumaire de Louis Bonaparte, Paris, Éd. J.-J. Pauvert,

Marx, Karl / Engels, Friedrich (1959 [1848]) : « Manifest der kommunistischen Partei », in : Marx, Karl / Engels Friedrich : Werke, vol. IV, Berlin.

Marx, Karl / Engels, Friedrich (1960 [1850]) : « Ansprache der Zentralbehörde an den Bund vom März 1850 », in : Marx, Karl / Engels Friedrich : Werke, vol. VII, Berlin.

Marx, Karl / Engels, Friedrich (1963) : Werke, vol. XXVIII, Berlin.

Mohl, Robert von (1855) : Die Geschichte und Literatur der Staatswissenschaften, vol. I., Erlangen.

Mommsen, Wilhelm (1960) : Deutsche Parteiprogramme, Munich.

Müller, Hans (1967) : Ursprung und Geschichte des Wortes « Sozialismus » und seiner Verwandten, Hanovre.

Naumann, Friedrich (1964 [1910]) : « Fortschrittliche Volkspartei!», in : Werke, vol. V, Cologne.

Roscher, Wilhelm (1847/1848) : «Umrisse zur Naturlehre der drei Staatsformen », in : Zeitschrift für Geschichtswissenschaft, 7 (1847), p. 79-87, p. 322-365, p. 436-473 ; 9 (1848), p. 285-325, p. 381-414.

Rotteck, Karl von / Welcker, Carl (éd.) (1837) : Staats-Lexikon oder Encyclopädie der Staatswissenschaften, vol. IV, Altona.

Rotteck, Karl von / Welcker, Carl (éd.) (1859) : Staats-Lexikon oder Encyclopädie der Staatswissenschaften, $3^{e}$ éd., vol. IV, Altona.

Schieder, Wolfgang (1963) : Anfänge der deutschen Arbeiterbewegung, Stuttgart.

Schlegel, Friedrich (1966 [1796]) : «Versuch über den Begriff des Republikanismus », in : Sämmtliche Werke, vol. VII, Munich.

Schleiermacher, Friedrich Daniel (1838 [1818]) : « Über die Begriffe der verschiedenen Staatsformen », in : Sämmtliche Werke, Abteilung III: Zur Philosophie, vol. II, Berlin, p. 246-286.

Seier, Hellmut (1961) : Die Staatsidee Heinrich von Sybels in den Wandlungen der Reichsgründungszeit 1862/71, Lübeck / Hambourg.

Srbik, Heinrich von (1925) : Metternich. Der Staatsmann und der Mensch, vol. I, Munich.

Stahl, Friedrich Julius (1863) : Die gegenwärtigen Parteien in Staat und Kirche, Berlin.

Stammhammer, Josef (1912) : Bibliographie der Social-Politik, vol. II, Iéna.

Stein, Lorenz von (1921) : Geschichte der sozialen Bewegung in Frankreich, réimp., Munich.

Stenographische Berichte über die öffentlichen Verhandlungen des Untersuchungsausschusses der verfassunggebenden deutschen Nationalversammlung, vol. III, 1848.

Stenographische Berichte über die öffentlichen Verhandlungen des Untersuchungsausschusses der verfassunggebenden deutschen Nationalversammlung, vol. IX, 1849.

Tocqueville, Alexis de (1951) : De la démocratie en Amérique, in : Cuvres complètes, vol. I.1 et I.2, Paris.

Trautmann, Günter (1968) : Wahlorganisation und parlamentarische Tätigkeit des Allgemeinen Arbeitervereins von 1867 bis 1871/74, Heidelberg. 
Treitschke, Heinrich von (1897) : Politik, éd. par Max Cornicelius, $2^{\mathrm{e}}$ éd., vol. I, Leipzig.

Treitschke, Heinrich von (1898) : Politik, éd. par Max Cornicelius, $2^{\mathrm{e}}$ éd., vol. II, Leipzig.

Wagener, Herrmann (1861) : Staats- und Gesellschaftslexikon, vol. VI, Berlin.

Wentzke, Paul / Klötzer, Wolfgang (éd.) (1959) : Deutscher Liberalismus im Vormärz. Heinrich v.

Gagern, Briefe und Reden 1815-1848, Göttingen.

Winter, Georg (éd.) (1931) : Die Reorganisation des Preußischen Staates unter Stein und Hardenberg, vol. I.1, Leipzig.

Zachariä, Karl Salomo (1839) : Vierzig Bücher vom Staate, $2^{\mathrm{e}}$ éd., vol. III, Heidelberg.

Ziegler, Franz Wilhelm (1850) : Wie ist dem Handwerkerstande zu helfen?, Berlin.

\section{NOTES}

296. Guizot (1849), p. 9-10.

297. Görres (1929 [1819]), p. 111 sq.

298. Ibid., p. 112.

299. Ibid., p. 135.

300. Arndt (1908 [1814]), p. 222.

301. Aretin / Rotteck (1838-1840). Une comparaison des deux éditions et de leur préface respective révèle, en passant d'Aretin à Rotteck, un déplacement de l'attention de l'aristocratie vers la démocratie.

302. Aretin / Rotteck (1838), p. 151 sq. ; de même, voir aussi p. 89.

303. Rotteck, article "Demokratisches Prinzip», in: Rotteck / Welcker (1837), p. 256: «Le principe monarchique et le principe démocratique peuvent tout à fait coexister, ils peuvent même se soutenir mutuellement ".

304. Ibid., p. 256.

305. Heinrich von Gagern, lettre à Max von Gagern, 15 mars 1838, in : Wentzke / Klötzer (1959), p. 196.

306. Hansemann (1834), p. 226-227 et p.228-229. Cf. la description de la Prusse comme «autocratie démocratique » (Immanuel Ogienski, zit. in: Hallische und deutsche Jahrbücher für Wissenschaft und Kunst, 4, 1841, p. 281).

307. Hardenberg, Rigaer Denkschrift (1807), in : Winter (1931), p. 306.

308. Gentz (1844 [1819]), p. 221-222.

309. Des témoignages sur ce point figurent chez Srbik (1925), p. 366-367.

310. «Denkschrift eines preußischen Staatsmanns aus dem Jahre 1822 », in : Klüber / Welcker (1844), p. 365.

311. Humboldt (1936), p. 293.

312. Schleiermacher (1838 [1818]), p. 246 sq., en particulier p. 268 et p. 271.

313. Voir Schlegel (1966 [1796]).

314. Brockhaus (1818), p. 305.

315. Brockhaus (1838), p. 914-915.

316. Eckermann (1988), p. 232 (15 juillet 1827).

317. Hegel (2013), p. 511-512; p. 507.

318. Ibid., p. 302 ; p. 462.

319. Hegel (1930 [1831]), p. 503.

320. Hegel (2013), p. 465-466.

321. Ibid., p. 476. 
322. Rotteck / Welcker (1837), p. 241 sq. et p. 252 sq.

323. Zachariä (1839), p. 230 sq., en particulier p. 231.

324. Friedrich Julius Stahl ([1863], p. 122) parlait de «l'appellation curieuse de "monarchie démocratique" ".

325. Bluntschli / Brater (1857), p. 689.

326. Rotteck / Welcker (1859), p. 357, article « Demokratie ».

327. Dahlmann (1835), p. 109 sq.

328. Gervinus (1853), p. 19 et p. 42. Sur la relation entre démocratie et protestantisme chez Gervinus, voir supra.

329. Ibid., p. 174 sq.

330. Mohl, dans : Kritische Zeitschrift Für Rechtswissenschaft und Gesetzgebung des Auslandes, 7, 1835 ; cit. in : Franz (1958), p. 87, accompagné d'autres témoignages. Sur l'application moderne de l'idée de représentation à la domination du peuple [Volksherrschaft], voir également Mohl (1855), p. 514.

331. Voir Mohl, recension de Tocqueville, De la démocratie en Amérique, in : Kritische Zeitschrift Für Rechtswissenschaft und Gesetzgebung des Auslandes, 8, 1836, p. 359 sq. et 16, 1844, p. 275 sq. Voir aussi Eschenburg (1959), p. XIX sq.

332. Tocqueville (1951), introduction, vol. I.1, p. 1-2 et p. 4.

333. Ibid., p. 45 sq.

334. Sur ce thème fondamental, fréquemment répété, voir notamment Tocqueville (1951), (II, 4, 6), vol. I.2, p. $322 \mathrm{sq}$.

335. Ibid. (I, 4, 7), p. 328.

336. Elysard (1842), p. 986.

337. Marr (1846), p. 115 et p. 172.

338. Fröbel (1847), p. 66 sq. et p. 468-469.

339. Brockhaus (1840), vol. III, p. 372 et vol. VIII, p. 833.

340. Pour plus de détails sur ce point, voir la thèse en cours d'achèvement de Hans Dreher, Radikale und soziale Demokratie am Ausgang der Juli-Monarchie, Heidelberg.

341. Duden (1963), p. 547.

342. Arnold Ruge, Deutsche Jahrbücher für Wissenschaft und Kunst, 12, 1843, p. 2.

343. Voir par exemple les choix linguistiques de Bauer (1847), qui utilise la plupart du temps le terme « radical », mais aussi ceux de " parti du mouvement » et de « démocratique ».

344. Voir par exemple la question portée par le député Jahn à l'Assemblée nationale de Francfort le 26 juillet, qui demande «si le pouvoir impérial ne veut pas prendre des mesures résolues contre les activités d'agitation des associations communistes de ceux que l'on nomme "radicauxdémocrates", [...] qui forment une conspiration contre l'ordre, la loi et la liberté et préparent une guerre civile sanglante » (Stenographische Berichte [1848], p. 1719).

345. Bauer (1847), vol. III, p. 27 (cf. Hess [1961], p. 197).

346. Voir infra : «3. La "démocratie sociale" » et «4. Marx et Engels ».

347. Député Simon, Stenographische Berichte (1849), p. 6419 et p. 6424.

348. Schwäbische Kronik, 7 juillet 1848. Même principe dans les statuts des associations patriotiques du Wurtemberg : Der Beobachter, 3 août 1848, cité in : Boldt (1970), p. 258.

349. Tract « Gegen Demokraten helfen nur Soldaten », Berlin, fin novembre 1848.

*. * N.d.T.: Dans l'espace germanophone, en particulier au temps de la Confédération germanique, le terme renvoie aux États de puissance intermédiaire qui cherchèrent à pratiquer, parallèlement à l'Autriche et à la Prusse, une politique commune. Nous pouvons citer, parmi ceux-ci, les royaumes de Bavière, de Hanovre, de Saxe ou de Wurtemberg.

350. Ces deux termes sont utilisés à plusieurs reprises dans les associations de travailleurs allemandes depuis 1834/1835. Sur ce point, voir Schieder (1963), p. 174 sq.

351. La notion apparaît pourtant dès 1838 chez Brockhaus (1838), p. 914-915.

352. Stammhammer (1912), p. 82. 
353. Voir supra, § $14 \mathrm{sq}$.

354. Sur ce point, voir Dreher (cf. supra, note 340 ).

355. Engels (1957 [1844]), p. 592 ; voir aussi Engels (1959 [1845/46]), p. 612.

356. Grün (1845), p. 82.

357. Stephan Born, Verbrüderung, 23 janvier 1849, cité in : Balser (1965), vol. I, p. 144, note 362 (note 361 pour la référence liée à Hecker).

358. Plusieurs références figurent chez Müller (1967), p. 157-158.

359. Stein (1921), vol. I, p. 122-123. Les citations suivantes proviennent du même passage.

360. Tocqueville parlait déjà d'un «État social démocratique » pour les États-Unis (voir exemple [1951], [2, 4, 6], vol. I.2, p. 322).

361. Cf. l'interprétation de Böckenförde (1963), p. 248 sq.

362. Stein (1921), vol. III, p. 207. Il poursuit sa réflexion dans l'article «Démocratie et aristocratie ", in: Brockhaus (1845), p. 306 sq. Il distingue ici la "démagogie sociale» de la "démocratie sociale», souhaitable dans le cadre du "parti constitutionnel », en vertu de la complémentarité du principe de mouvement (la démocratie) et du principe de préservation (l'aristocratie) (cf. article « Noblesse »).

363. Müller (1967), p. 162.

364. Vorbote, $9,1867$.

365. Nürnberger Anzeiger (1869), cité in : Eckert (1968), p. 122-123.

366. «Gedanken über die moderne schöne Literatur », Deutsche Vierteljahrsschrift, 3, 1840, p. 269.

367. Häusser (1851), p. 17.

368. Ziegler (1850), cité in : Balser (1965), vol. II, p. 569.

369. Marx (1975), p. 56 ; p. 68-70.

370. Engels (1964 [1843]), p. 481.

371. Engels (1959 [1845/46]), p. 613.

372. Sur ce point, voir par exemple Engels (1959 [1847]a), p. 316-317 ; (1959 [1847]b), p. 372-373 ; Marx / Engels (1959 [1848]), p. 481-482 et p. 492-493.

373. Marx / Engels (1960 [1850]), p. 244 sq., en particulier p. 244 et p. 246.

374. Même si les "jacobins" allemands avant 1800, les "démocrates" vers 1848 et Marx et Engels, de manière plus radicale, aspiraient à une " république une et indivisible », le centralisme unitaire n'appartenait pas nécessairement, même pour eux, au concept de démocratie; par contraste avec cette tendance, il était souvent explicitement lié au fédéralisme, pour lequel les États-Unis et la Suisse fournissaient des modèles.

375. Marx / Engels (1960 [1850]), p. 254.

376. Ibid., p. 248.

377. Marx (1964), p. 260.

378. Engels évoque les "purs démocrates » dans une lettre à Joseph Weydemeyer, en date du 12 avril 1853 (Marx / Engels [1963], p. 579).

379. Voir par exemple Marx (1962 [1875]), p. 15 sq.

380. Lassalle (1919b), p. 273. Une nouvelle interprétation de la pensée politique de Lassalle est développée dans la thèse de Trautmann (1968).

381. Lassalle (1919a), p. 31-32: « Le principe des nationalités libres et indépendantes est donc la base et la source, le père et la racine du concept de démocratie en général ».

382. Naumann (1964 [1910]), p.448. Cf. le programme du Parti populaire allemand [Deutsche Volkspartei] en 1895 (Mommsen [1960], p. 160 sq).

383. Wagener (1861), p. 112.

384. Seier (1961), p. 22-23.

*. * N.d.T. : La notion renvoie, dans le contexte des réformes prussiennes du XIX ${ }^{\mathrm{e}}$ siècle, à la volonté de renforcer l'autonomie des communes vis-à-vis de l'autorité centrale, notamment en matière de finances. Conçu en opposition à l'organisation des communes en France, le modèle de 
la Selbstverwaltung se veut aussi, durant cette période, un retour à certaines traditions médiévales.

385. Ibid., p. 53 ; Treitschke (1897), p. 161.

386. Roscher (1847/1848). Sur ce point, voir Bussmann (1952), p. 181 sq.

387. Bussmann (1952), p. 195.

388. Treitschke (1898), p. 257.

389. Ibid., p. 9.

390. Ibid., passim, en particulier, p. 158-159.

391. Ibid., p. 160-161.

392. Cité in : Bussmann (1952), p. 231-232.

393. Jellinek (1960 [1900]), p. 666 et p. 710 sq.

394. Chez Wichern, le mot même de "démocratie " est à peine utilisé. Voir une des rares déclarations de Wichern, tout à fait péremptoire, chez Gerhardt (1931), vol. III, p. 246. Ketteler souligna pour sa part à maintes reprises que l'esprit d'un État sécularisé ou anti-chrétien et le christianisme étaient incompatibles. Il était totalement éloigné du concept de "démocratie chrétienne ». Cf. Ketteler (1924), passim, en particulier vol. I, p. 247 sq. et vol. II, p. 5 sq.

395. Voir Maier (1965) et (1964), p. 11 sq. L'ouvrage de Michael Fogarty (1959) demeure pauvre sur le plan de l'histoire des concepts.

396. Bachem (1931) et (1932), passim. Six mois après la parution de l'encyclique Graves de communi, Lieber parlait de la "démocratie chrétienne " au Congrès des catholiques allemands (Osnabrück, août 1901) (voir Filthaut [1960], p. 164).

397. Bachem (1930), p. 300 et p. 316.

INDEX

Schlüsselwörter : Demokratie, konstitutionelle Monarchie

Mots-clés : démocratie, monarchie constitutionnelle

\section{AUTEURS}

\section{WERNER CONZE}

Werner Conze (1910-1986) a été professeur d'histoire à l'université de Heidelberg. Pour plus d'informations, voir la notice suivante. 